\title{
Aspterrics A and B, New Sesquiterpenes from Deep Sea-derived Fungus Aspergillus terreus YPGA10
}

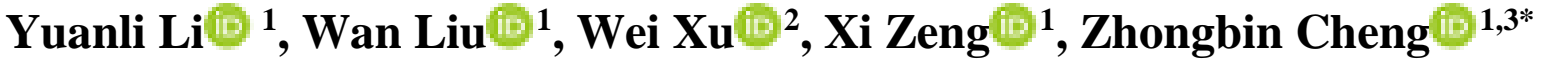 and Qin $\mathrm{Li}$ 1,3*}

\author{
${ }^{1}$ Pharmaceutical College, Henan University, Kaifeng 475004, People's Republic of China \\ ${ }^{2}$ Key Laboratory of Marine Biogenetic Resources, Third Institute of Oceanography, Ministry of \\ Natural Resources, Xiamen 361005, People's Republic of China \\ ${ }^{3}$ Eucommia ulmoides Cultivation and Utilization of Henan Engineering Laboratory, Kaifeng 475004, \\ People's Republic of China
}

(Received April 16, 2019; Revised May 13, 2019; Accepted May 14, 2019)

\begin{abstract}
Two new sesquiterpenes, namely aspterric A (1) and aspterric B (2), together with aspterric acid (3), were isolated from the deep-sea-derived fungus Aspergillus terreus YPGA10. The structures of aspterrics A and B were determined by NMR and HRESIMS data. Biogenetically, aspterrics A and B were assumed to be the intermediates to derive aspterric acid.
\end{abstract}

Keywords: Aspergillus terreus; deep-sea-derived fungus; sesquiterpenoids; aspterrics A and B. (C) 2019 ACG Publications. All rights reserved.

\section{Introduction}

The fungus Aspergillus terreus, widely distributes in natural environment, has proved to be a potential fungus for producing molecules with significant bioactivity or complex structures. Previous studies of this fungus have led to the identification of meroterpenoids [1,2], sesterterpenoids [3], butenolides [4-6], alkaloids [7], and cyclic peptides [8]. Some possess unique carbon skeletons and some exhibit remarkable bioactivities such as enzyme inhibition, antitumor activity, and antiviral activity. As part of our ongoing efforts to discover bioactive molecules from marine-derived fungi [911], chemical examination of a deep-sea sediment-derived Aspergillus terreus YPGA10 led to the isolation of a carotane-type sesquiterpene aspterric acid (3) and two new biogenetically related sesquiterpenes (1 and $\mathbf{2}$ ). Herein, the isolation and structural identification were described.

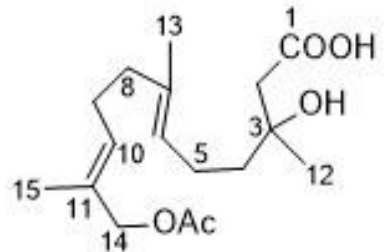

1

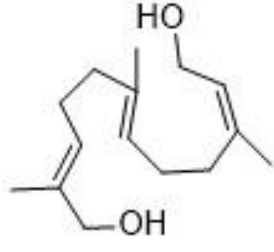

2

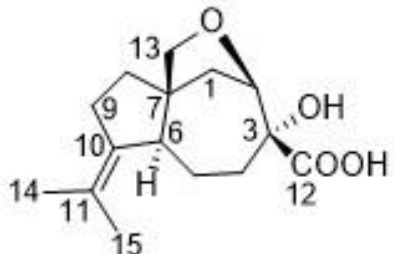

3

Figure 1. Structures of compounds 1-3 from Aspergillus terreus YPGA10

*Corresponding author: E-Mail: czb360@126.com; liqin6006@163.com. 


\section{Materials and Methods}

\subsection{Microorganism Material}

Fungus Aspergillus terreus (YPGA10) was isolated from the deep-sea sediment collected in the Yap Trench at a depth of $4159 \mathrm{~m}$. The strain was identified as Aspergillus terreus based on microscopic examination and by internal transcribed spacer (ITS) sequencing. The ITS sequence has been deposited in GenBank (http://www.ncbi.nlm.nih.gov) with accession number MG835907. The strain YPGA10 (MCCC 3A01013) was deposited at the Marine Culture Collection of China.

\subsection{Fermentation and Isolation}

The fermentation was conducted in 40 Fernbach flasks $(500 \mathrm{~mL})$, each containing $70 \mathrm{~g}$ of rice. Distilled $\mathrm{H}_{2} \mathrm{O}(90 \mathrm{~mL})$ was added and the contents were autoclaved at 15 psi for $30 \mathrm{~min}$. After cooling to room temperature (r.t.), each flask was inoculated with $3.0 \mathrm{~mL}$ of the spore inoculum and incubated at r.t. for 40 days. The fermented material was extracted with EtOAc $(4000 \mathrm{~mL})$ for three times. After evaporation under vacuum, the EtOAc extract $(12.0 \mathrm{~g})$ was chromatographed by ODS silica gel column chromatography $(\mathrm{CC})\left(\mathrm{MeOH} / \mathrm{H}_{2} \mathrm{O}: 20: 80 \rightarrow 100: 0\right)$ to give ten fractions (F1-F10). F7 (0.40 g) was subjected to HPLC on a semipreparative YMC-pack ODS-A column using $\mathrm{MeOH} / \mathrm{H}_{2} \mathrm{O}(65: 35,2$ $\mathrm{mL} / \mathrm{min}$ ) to obtain seven fractions (F7a-F7g). F7f was purified by HPLC eluted with $\mathrm{CH}_{3} \mathrm{CN} / \mathrm{H}_{2} \mathrm{O}$ $(57: 43,2 \mathrm{~mL} / \mathrm{min})$ to obtain $1\left(7.1 \mathrm{mg}, \mathrm{t}_{\mathrm{R}} 35.5 \mathrm{~min}\right)$ and $\mathbf{2}\left(2.8 \mathrm{mg}, \mathrm{t}_{\mathrm{R}} 39.5 \mathrm{~min}\right)$. F6 was subjected to Sephadex LH-20 CC using MeOH to give four fractions (F6a-F6d). F6a was separated on the YMC column with a mobile phase of $\mathrm{CH}_{3} \mathrm{CN} / \mathrm{H}_{2} \mathrm{O}(53: 47,2 \mathrm{~mL} / \mathrm{min})$ to afford $3\left(15 \mathrm{mg}, \mathrm{t}_{\mathrm{R}} 27.5 \mathrm{~min}\right)$.

Aspterric A (1): Colorless oil; $[\alpha]^{20}{ }_{\mathrm{D}} 0(c 0.05, \mathrm{MeOH}) ;{ }^{1} \mathrm{H}$ NMR (methanol- $\left.d_{4}, 400 \mathrm{MHz}\right) \delta_{\mathrm{H}} 2.45$ (2H, s, H $\left.{ }_{2}-2\right), 1.57$ (2H, m, H $\left.{ }_{2}-4\right), 2.09$ (2H, m, H $\left.2-5\right), 5.14(1 \mathrm{H}, \mathrm{t}, J=7.1 \mathrm{~Hz}, \mathrm{H}-6), 2.03\left(2 \mathrm{H}, \mathrm{m}, \mathrm{H}_{2}-\right.$ 8), $2.16\left(2 \mathrm{H}, \mathrm{m}, \mathrm{H}_{2}-9\right), 5.46(1 \mathrm{H}, \mathrm{t}, J=7.1 \mathrm{~Hz}, \mathrm{H}-10), 1.28\left(3 \mathrm{H}, \mathrm{s}, \mathrm{H}_{3}-12\right), 1.63\left(3 \mathrm{H}, \mathrm{s}, \mathrm{H}_{3}-13\right), 4.44$ $\left(2 \mathrm{H}, \mathrm{s}, \mathrm{H}_{2}-14\right), 1.65\left(3 \mathrm{H}, \mathrm{s}, \mathrm{H}_{3}-15\right), 2.04(3 \mathrm{H}, \mathrm{s}, \mathrm{COCH} 3) .{ }^{13} \mathrm{C}$ NMR (methanol- $\left.d_{4}, 100 \mathrm{MHz}\right) \delta_{\mathrm{C}} 175.9$ (C-1), 46.5 (C-2), 72.1 (C-3), 42.7 (C-4), 23.5 (C-5), 125.9 (C-6), 135.6 (C-7), 40.1 (C-8), 27.1 (C-9), 130.3 (C-10), 131.4 (C-11), 27.2 (C-12), 16.0 (C-13), 71.3 (C-14), 14.0 (C-15), $172.9\left(\underline{\mathrm{COCH}}_{3}\right), 20.8$ $\left(\mathrm{COCH} 3{ }_{3}\right)$. HRESIMS $m / z 311.1853[\mathrm{M}-\mathrm{H}]^{-}$(calcd for $\left.\mathrm{C}_{17} \mathrm{H}_{27} \mathrm{O}_{5}, 311.1864\right)$.

Aspterric B (2): Colorless oil; ${ }^{1} \mathrm{H}$ NMR (methanol- $\left.d_{4}, 400 \mathrm{MHz}\right) \delta_{\mathrm{H}} 4.06\left(2 \mathrm{H}, \mathrm{dd}, J=6.8,0.8 \mathrm{~Hz}, \mathrm{H}_{2}-\right.$ 1), $5.36(1 \mathrm{H}, \mathrm{br} \mathrm{t}, J=6.8 \mathrm{~Hz}, \mathrm{H}-2), 2.10\left(2 \mathrm{H}, \mathrm{m}, \mathrm{H}_{2}-4\right), 2.10\left(2 \mathrm{H}, \mathrm{m}, \mathrm{H}_{2}-5\right), 5.15(1 \mathrm{H}, \mathrm{t}, J=7.0 \mathrm{~Hz}, \mathrm{H}-$ 6), $2.02\left(2 \mathrm{H}, \mathrm{m}, \mathrm{H}_{2}-8\right), 2.14\left(2 \mathrm{H}, \mathrm{m}, \mathrm{H}_{2}-9\right), 5.39(1 \mathrm{H}, \mathrm{t}, J=7.0 \mathrm{~Hz}, \mathrm{H}-10), 1.75\left(3 \mathrm{H}, \mathrm{s}, \mathrm{H}_{3}-12\right), 1.63$ $\left(3 \mathrm{H}, \mathrm{s}, \mathrm{H}_{3}-13\right), 3.91\left(2 \mathrm{H}, \mathrm{s}, \mathrm{H}_{2}-14\right), 1.65\left(3 \mathrm{H}, \mathrm{s}, \mathrm{H}_{3}-15\right) .{ }^{13} \mathrm{C} \mathrm{NMR}$ (methanol- $\left.d_{4}, 100 \mathrm{MHz}\right) \delta_{\mathrm{C}} 59.2(\mathrm{C}-$ 1), 125.7 (C-2), 139.6 (C-3), 32.9 (C-4), 27.6 (C-5), 125.2 (C-6), 136.3 (C-7), 40.5 (C-8), 27.3 (C-9), 126.5 (C-10), 135.9 (C-11), 23.7 (C-12), 16.1 (C-13), 68.9 (C-14), 13.7 (C-15). HRESIMS m/z [M + $\mathrm{Na}]^{+} 261.1826$ (calcd for $\mathrm{C}_{15} \mathrm{H}_{26} \mathrm{O}_{2} \mathrm{Na}^{+}, 261.1825$ ).

Aspterric acid (3): Colorless oil; ${ }^{13} \mathrm{C}$ NMR (methanol- $\left.d_{4}, 100 \mathrm{MHz}\right) \delta_{\mathrm{C}} 35.2(\mathrm{C}-1), 84.8(\mathrm{C}-2), 76.4$ (C-3), 33.1 (C-4), 36.7 (C-5), 56.7 (C-6), 54.2 (C-7), 24.8 (C-8), 35.0 (C-9), 136.7 (C-10), 125.1 (C11), 177.7 (C-12), 79.4 (C-13), 21.0 (C-14), 23.4 (C-15).

\section{Results and Discussion}

The molecular formula of compound $\mathbf{1}$ was determined to be $\mathrm{C}_{17} \mathrm{H}_{28} \mathrm{O}_{5}$ by the HRESIMS data, requiring four degrees of unsaturation. The ${ }^{1} \mathrm{H}$ NMR and HSQC spectra displayed signals for four methyl singlets $\left[\delta_{\mathrm{H}} 1.28\left(3 \mathrm{H}, \mathrm{s}, \mathrm{H}_{3}-12\right), 1.65\left(3 \mathrm{H}, \mathrm{s}, \mathrm{H}_{3}-13\right), 1.67\left(3 \mathrm{H}, \mathrm{s}, \mathrm{H}_{3}-15\right), 2.04(3 \mathrm{H}, \mathrm{s}\right.$, $\left.\mathrm{COCH}_{3}\right)$ ], two olefinic protons [ $\left.\delta_{\mathrm{H}} 5.14(1 \mathrm{H}, \mathrm{t}, J=7.1 \mathrm{~Hz}, \mathrm{H}-6), 5.46(1 \mathrm{H}, \mathrm{t}, J=7.1 \mathrm{~Hz}, \mathrm{H}-10)\right]$, and six methylenes $\left[\delta_{\mathrm{H}} 2.45\left(2 \mathrm{H}, \mathrm{s}, \mathrm{H}_{2}-2\right), 1.57\left(2 \mathrm{H}, \mathrm{m}, \mathrm{H}_{2}-4\right), 2.09\left(2 \mathrm{H}, \mathrm{m}, \mathrm{H}_{2}-5\right), 2.03\left(2 \mathrm{H}, \mathrm{m}, \mathrm{H}_{2}-8\right)\right.$, $\left.2.16\left(2 \mathrm{H}, \mathrm{m}, \mathrm{H}_{2}-9\right), 4.44\left(2 \mathrm{H}, \mathrm{s}, \mathrm{H}_{2}-14\right)\right]$. The ${ }^{13} \mathrm{C}$ NMR spectrum exhibited a total of 17 carbon resonances, including four olefinic carbons $\left[\delta_{\mathrm{C}} 125.9,135.6,130.3,131.4\right]$ for two double bonds, a 
carbonyl carbon $\left[\delta_{\mathrm{C}} 172.9\left(\mathrm{COCH}_{3}\right)\right]$ for an acetyl carbonyl carbon, and a carboxylic acid group $\left[\delta_{\mathrm{C}}\right.$ 175.9 (C-1)]. The two carbonyl carbons and two double bonds accounted for all four degrees of unsaturation, indicating 1 to be acyclic. The structure was further established to be a new farnesol derivative by $2 \mathrm{D}$ NMR data (Figure 2$)$. The HMBC correlations from $\mathrm{H}_{3}-12\left(\delta_{\mathrm{H}} 1.28\right)$ to $\mathrm{C}-2\left(\delta_{\mathrm{C}} 46.5\right)$, $\mathrm{C}-3\left(\delta_{\mathrm{C}} 72.1\right), \mathrm{C}-4\left(\delta_{\mathrm{C}} 42.7\right)$ and $\mathrm{H}_{2}-2\left(\delta_{\mathrm{H}} 2.45\right)$ to the carboxylic acid carbon $\left(\delta_{\mathrm{C}} 175.9\right)$ established a 3-hydroxy-3-methylbutanoic acid moiety (unit A). The COSY relationship from $\mathrm{H}_{2}-5\left(\delta_{\mathrm{H}} 2.09\right)$ to $\mathrm{H}-6$ $\left(\delta_{\mathrm{H}} 5.14\right)$ and $\mathrm{HMBC}$ correlations from $\mathrm{H}_{3}-13\left(\delta_{\mathrm{H}} 1.63\right)$ to $\mathrm{C}-6\left(\delta_{\mathrm{C}} 125.9\right), \mathrm{C}-7\left(\delta_{\mathrm{C}} 135.6\right)$, and $\mathrm{C}-8\left(\delta_{\mathrm{C}}\right.$ 40.1) established an isopentenyl unit (unit B). Additional HMBC correlations from $\mathrm{H}_{2}-14\left(\delta_{\mathrm{H}} 4.44\right)$ to $\mathrm{C}-10\left(\delta_{\mathrm{C}} 130.3\right), \mathrm{C}-11\left(\delta_{\mathrm{C}} 131.4\right), \mathrm{C}-15\left(\delta_{\mathrm{C}} 14.0\right)$ and the COSY relationship between $\mathrm{H}_{2}-9\left(\delta_{\mathrm{H}} 2.16\right)$ and $\mathrm{H}-10\left(\delta_{\mathrm{H}} 5.46\right)$ established another isopentenyl unit (Unit $\left.\mathrm{C}\right)$. The above three units were connected by the COSY correlations from $\mathrm{H}_{2}-8$ to $\mathrm{H}_{2}-9$ and from $\mathrm{H}_{2}-4$ to $\mathrm{H}_{2}-5$. The remaining acetyl group was located at $\mathrm{C}-14\left(\delta_{\mathrm{C}} 71.3\right)$ by the $\mathrm{HMBC}$ correlation from $\mathrm{H}_{2}-14$ to the acetyl carbonyl carbon $\left(\delta_{\mathrm{C}} 172.9\right)$. The strong NOESY correlations of $\mathrm{H}_{3}-13 / \mathrm{H}_{2}-5, \mathrm{H}_{2}-8 / \mathrm{H}-6, \mathrm{H}_{3}-15 / \mathrm{H}_{2}-9$, and $\mathrm{H}_{2}-$ $14 / \mathrm{H}-10$ indicated that both $\Delta^{6}$ and $\Delta^{10}$ had an $E$ configuration. The specific rotation of $1\left([\alpha]^{20}{ }_{\mathrm{D}} 0\right)$ indicated that 1 was racemic. Compound 1 was given the trivial name aspterric A.

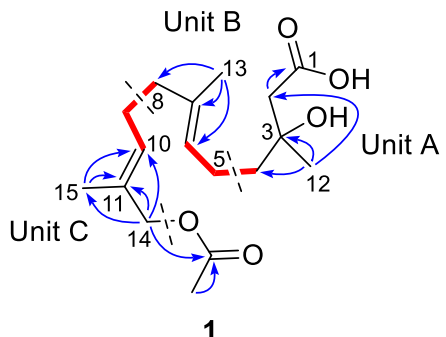

1

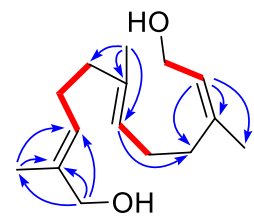

2

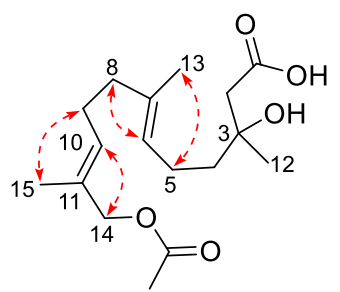

1

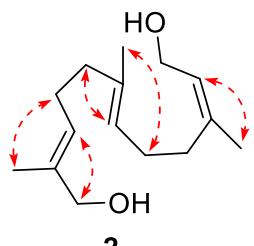

Figure 2. ${ }^{1} \mathrm{H}-{ }^{1} \mathrm{H} \operatorname{COSY}(-), \operatorname{HMBC}(\smile)$, and $\operatorname{NOE}\left({ }^{*}-\pi\right)$ correlations of $\mathbf{1}$ and 2.

Compound 2 had the molecular formula $\mathrm{C}_{15} \mathrm{H}_{26} \mathrm{O}_{2}$ based on the HRESIMS and NMR data, indicating three degrees of unsaturation. The ${ }^{1} \mathrm{H}$ NMR and HSQC spectra displayed the signals for three three olefinic protons $\left[\delta_{\mathrm{H}} 5.36(1 \mathrm{H}, \mathrm{t}, J=6.8 \mathrm{~Hz}, \mathrm{H}-2), 5.15(1 \mathrm{H}, \mathrm{t}, J=7.0 \mathrm{~Hz}, \mathrm{H}-6), 5.39(1 \mathrm{H}, \mathrm{t}\right.$, $J=7.0 \mathrm{~Hz}, \mathrm{H}-10)]$, three olefinic methyls $\left[\delta_{\mathrm{H}} 1.63\left(3 \mathrm{H}, \mathrm{s}, \mathrm{H}_{3}-13\right), 1.65\left(3 \mathrm{H}, \mathrm{s}, \mathrm{H}_{3}-15\right), 1.75\left(3 \mathrm{H}, \mathrm{s}, \mathrm{H}_{3}-\right.\right.$ 12)], and six methylenes [ $\delta_{\mathrm{H}} 4.06\left(2 \mathrm{H}, \mathrm{dd}, J=6.8,0.8 \mathrm{~Hz}, \mathrm{H}_{2}-1\right), 2.10\left(2 \mathrm{H}, \mathrm{m}, \mathrm{H}_{2}-4\right), 2.10\left(2 \mathrm{H}, \mathrm{m}, \mathrm{H}_{2}-\right.$ 5), $\left.2.02\left(2 \mathrm{H}, \mathrm{m}, \mathrm{H}_{2}-8\right), 2.14\left(2 \mathrm{H}, \mathrm{m}, \mathrm{H}_{2}-9\right), 3.91\left(2 \mathrm{H}, \mathrm{s}, \mathrm{H}_{2}-14\right)\right]$. The ${ }^{13} \mathrm{C}$ NMR spectrum, in combination with HSQC spectrum, resolved 15 carbon resonances attributable to three double bonds, three methyls, and six methylenes. The aforementioned data were similar to those of $\mathbf{1}$, indicating a farnesol derivative [12]. The structure was further established by detailed 2D NMR analyses (Figure 2). The HMBC and COSY correlations established the same units $B$ and $C$ as those of $\mathbf{1}$, while unit $A$ was determined to be an isopentenyl alcohol moiety by the HMBC correlations from the additional olefinic proton $\mathrm{H}-2\left(\delta_{\mathrm{H}} 5.36\right)$ to $\mathrm{C}-3\left(\delta_{\mathrm{C}} 139.6\right), \mathrm{C}-4\left(\delta_{\mathrm{C}} 32.9\right)$, and $\mathrm{C}-12\left(\delta_{\mathrm{C}} 23.7\right)$ and between its COSY relationship with the extra oxygenated methylene protons $\mathrm{H}_{2}-1\left(\delta_{\mathrm{H}} 4.08\right)$. The three moieties were connected by the HMBC correlation from H-6 $\left(\delta_{\mathrm{H}} 5.15\right)$ to $\mathrm{C}-4\left(\delta_{\mathrm{C}} 32.9\right)$ and COSY relationship from $\mathrm{H}_{2}-8\left(\delta_{\mathrm{H}} 2.02\right)$ to $\mathrm{H}-9\left(\delta_{\mathrm{H}} 2.14\right)$. The strong NOESY correlations of $\mathrm{H}_{3}-13\left(\delta_{\mathrm{H}} 1.63\right) / \mathrm{H}_{2}-5\left(\delta_{\mathrm{H}}\right.$ $2.10), \mathrm{H}_{2}-8 / \mathrm{H}-6, \mathrm{H}_{3}-15\left(\delta_{\mathrm{H}} 1.65\right) / \mathrm{H}_{2}-9\left(\delta_{\mathrm{H}} 2.14\right), \mathrm{H}_{2}-14\left(\delta_{\mathrm{H}} 3.91\right) / \mathrm{H}-10\left(\delta_{\mathrm{H}} 5.39\right), \mathrm{H}_{2}-1\left(\delta_{\mathrm{H}} 4.08\right) / \mathrm{H}_{2}-4$ $\left(\delta_{\mathrm{H}} 2.10\right), \mathrm{H}-2\left(\delta_{\mathrm{H}} 5.36\right) / \mathrm{H}_{3}-12\left(\delta_{\mathrm{H}} 1.75\right)$ indicated that both $\Delta^{6}$ and $\Delta^{10}$ had an $E$ configuration, while $\Delta^{2}$ had a $Z$ configuration. Compound 2 was named aspterric B.

Compound 3 was identified to be aspterric acid by careful comparison with NMR data and specific rotation reported in the literature [13]. 


\section{Conclusion}

Three sesquiterpenoids including two new farnesol derivatives were isolated from the deepsea-derived fungus Aspergillus terreus YPGA10. The structures of aspterrics A and B were established by detailed analyses of the NMR data and HRESIMS data. Biogenetically, aspterrics A and $\mathrm{B}$ were assumed to be the intermediates to derive aspterric acid.

\section{Acknowledgments}

This work was supported by the grants from China Postdoctoral Science Foundation (2018M630815), China Ocean Mineral Resources R\&D Association (COMRA) Program (DY135-B209), National Key R\&D Program of China (2017YFD0600702-2), and Start-Up Funding of Henan University (B2017067).

\section{Supporting Information}

Supporting information accompanies this paper on http://www.acgpubs.org/journal/records-ofnatural-products

\section{ORCID}

Yuanli Li:0000-0001-6953-720X

Wan Liu:0000-0001-5612-9434

Wei Xu:0000-0002-3265-7475

Xi Zeng:0000-0001-8742-1390

Zhongbin Cheng: 0000-0003-0942-6422

Qin Li:0000-0001-8295-6230

\section{References}

[1] C. Qi, M. Liu, Q. Zhou, W. Gao, C. Chen, Y. Lai, Z. Hu, Y. Xue, J. Zhang, D. Li, X.-N. Li, Q. Zhang, J. Wang, H. Zhu and Y. Zhang (2018). BACE1 inhibitory meroterpenoids from Aspergillus terreus, J Nat. Prod. 81, 1937-1945.

[2] C.-C. Liaw, Y.-L. Yang, C.-K. Lin, J.-C. Lee, W.-Y. Liao, C.-N. Shen, J.-H. Sheu and S.-H. Wu (2015). New meroterpenoids from Aspergillus terreus with inhibition of cyclooxygenase-2 expression, Org. Lett. 17, 2330-2333.

[3] Z. Liu, Y. Chen, S. Chen, Y. Liu, Y. Lu, D. Chen, Y. Lin, X. Huang and Z. She (2016). Aspterpenacids A and B, two sesterterpenoids from a mangrove endophytic fungus Aspergillus terreus H010, Org. Lett. 18, 1406-1409.

[4] Y. Sun, J. Liu, L. Li, C. Gong, S. Wang, F. Yang, H. Hua and H. Lin (2018). New butenolide derivatives from the marine sponge-derived fungus Aspergillus terreus, Bioorg. Med. Chem. Lett. 28, 315-318.

[5] K. Sun, G. Zhu, J. Hao, Y. Wang and W. Zhu (2018). Chemical-epigenetic method to enhance the chemodiversity of the marine algicolous fungus, Aspergillus terreus OUCMDZ-2739, Tetrahedron 74, 83-87.

[6] C. Qi, W. Gao, D. Guan, J. Wang, M. Liu, C. Chen, H. Zhu, Y. Zhou, Y. Lai, Z. Hu, Q. Zhou and Y. Zhang (2018). Butenolides from a marine-derived fungus Aspergillus terreus with antitumor activities against pancreatic ductal adenocarcinoma cells, Bioorg. Med. Chem. 26, 5903-5910.

[7] S. Cai, L. Du, A.L. Gerea, J.B. King, J. You and R.H. Cichewicz (2013). Spiro fused diterpene-indole alkaloids from a creek-bottom-derived Aspergillus terreus, Org. Lett. 15, 4186-4189.

[8] F. He, J. Bao, X. Y. Zhang, Z. C. Tu, Y. M. Shi and S. H. Qi (2013). Asperterrestide A, a cytotoxic cyclic tetrapeptide from the marine-derived fungus Aspergillus terreus SCSGAF0162, J Nat. Prod. 76, $1182-1186$.

[9] L. Liu, W. Xu, S. Li, M. Chen, Y. Cheng, W. Yuan, Z. Cheng and Q. Li (2018). Penicindopene A, a new indole diterpene from the deep-sea fungus Penicillium sp. YPCMAC1, Nat. Prod. Res. Doi:10.1080/14786419.2018.1514402. 
[10] Z. Cheng, W. Xu, L. Liu, S. Li, W. Yuan, Z. Luo, J. Zhang, Y. Cheng and Q. Li (2018). Peniginsengins B-E, new farnesylcyclohexenones from the deep sea-derived fungus Penicillium sp. YPGA11, Mar. Drugs 16, E358.

[11] Z. Cheng, W. Xu, Y. Wang, S. Bai, L. Liu, Z. Luo, W. Yuan and Q. Li (2019). Two new meroterpenoids and two new monoterpenoids from the deep sea-derived fungus Penicillium sp. YPGA11, Fitoterapia 133, 120-124.

[12] P. Lai, H. Rao and Y. Gao (2018). Chemical composition, cytotoxic, antimicrobial and antioxidant activities of essential oil from Anthriscus caucalis M. Bieb grown in China, Rec. Nat. Prod. 12, 290294.

[13] A. Shimada, M. Kusano, S. Takeuchi, S. Fujioka, T. Inokuchi and Y. Kimura (2002). Aspterric acid and 6-hydroxymellein, inhibitors of pollen development in Arabidopsis thaliana, produced by Aspergillus terreus, Z. Naturforsch. C 57, 459-464.

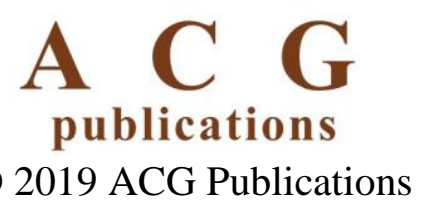

